\title{
Dasatinib, a small molecule inhibitor of the Src kinase, reduces the growth and activates apoptosis in pre-neoplastic Barrett's esophagus cell lines: Evidence for a noninvasive treatment of high-grade dysplasia
}

\author{
Landon J. Inge, PhD, ${ }^{\mathrm{a}}$ Aaron J. Fowler, BA, ${ }^{\mathrm{a}}$ Kimberly M. Paquette, MS, ${ }^{\mathrm{b}}$ Amanda L. Richer, BS, ${ }^{\mathrm{a}}$
} Nhan Tran, $\mathrm{PhD}{ }^{\mathrm{b}}$ and Ross M. Bremner, MD, $\mathrm{PhD}^{\mathrm{a}}$

\begin{abstract}
Background: Only local ablation (radiofrequency ablation, cryotherapy) or esophagectomy currently is available to treat high-grade dysplasia in Barrett's esophagus. Alternative treatments, specifically chemopreventive strategies, are lacking. Our understanding of the molecular changes of high-grade dysplasia in Barrett's esophagus offers an opportunity to inhibit neoplastic progression of high-grade dysplasia in Barrett's esophagus. Increased activity of the Src kinase and deregulation of the tumor suppressor p27 are features of malignant cells and high-grade dysplasia in Barrett's esophagus. Src phosphorylates p27, inhibiting its regulatory function and increasing cell growth and proliferation. We hypothesized that a small molecule inhibitor of Src might reduce the growth and reverse Src-mediated deregulation of p27 in Barrett's esophagus cells.
\end{abstract}

Methods: Immortalized Barrett's esophagus cell lines established from patient biopsies were treated with the Src kinase inhibitor dasatinib and evaluated for p27 localization and protein levels, as well as for effects on the cell cycle and apoptosis using flow cytometry, viability assays, and protein and RNA markers.

Results: Dasatinib reduced both Src activation and p27 phosphorylation and increased p27 protein levels and nuclear localization. These effects correlated with decreased proliferation, cell-cycle arrest, and activation of apoptosis. Analysis of biopsies of patients with Barrett's esophagus revealed the presence of phosphorylated p27 in high-grade dysplasia, consistent with in vitro findings.

Conclusions: Dasatinib has considerable antineoplastic effects on Barrett's esophagus cell lines carrying genetic markers associated with dysplasia, which correlates with the reversal of p27 deregulation. These findings suggest that dasatinib has potential as a treatment for patients with high-grade dysplasia and Barrett's esophagus and that p27 holds promise as a biomarker in the clinical use of dasatinib in patients with high-grade dysplasia and Barrett's esophagus. (J Thorac Cardiovasc Surg 2013;145:531-8)

Barrett's esophagus (BE) is the primary risk factor for esophageal adenocarcinoma, one of the fastest growing malignancies in the Western world. $^{1,2}$ Progression to early adenocarcinoma in nondysplastic $\mathrm{BE}$ is low $\left(0.5 \%-1 \%\right.$ per year). ${ }^{1}$ However, this risk increases to $7.4 \%$ per year in BE with high-grade dysplasia (HGD), with invasive cancer developing in 5 years in $50 \%$ of patients with HGD-BE. ${ }^{3,4}$ This increased risk has led to the

\footnotetext{
From the Center for Thoracic Disease and Transplantation, ${ }^{\mathrm{a}}$ Heart and Lung Institute, St Joseph's Hospital and Medical Center, Phoenix, Ariz; and Cancer and Cell

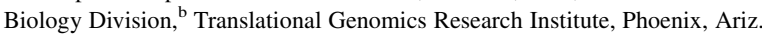
This work was funded by a grant from the St Joseph's Foundation (Phoenix, Ariz). Disclosures: Authors have nothing to disclose with regard to commercial support. L.J.I. and A.J.F. contributed equally to this work.

Read at the 38th Annual Meeting of The Western Thoracic Surgical Association, Maui, Hawaii, June 27-30, 2012.

Received for publication June 19, 2012; revisions received Oct 2, 2012; accepted for publication Oct 22, 2012; available ahead of print Nov 12, 2012

Address for reprints: Landon J. Inge, $\mathrm{PhD}$, Heart and Lung Institute, St Joseph's Hospital and Medical Center, 445 N 5th St, Suite 110, Phoenix, AZ 85004 (E-mail: landon.inge@dignityhealth.org).

0022-5223/ $\$ 36.00$

Copyright (C) 2013 by The American Association for Thoracic Surgery http://dx.doi.org/10.1016/j.jtcvs.2012.10.041
}

current treatment paradigm for BE, specifically, annual or biannual endoscopic surveillance for HGD and removal of HGD lesions by endoscopic resection, radiofrequency/ cryoablation, or esophagectomy. ${ }^{5}$ The invasive nature of these procedures has precipitated investigations into chemopreventive targets and agents as alternatives to noninvasively treat or control progression of HGD-BE. ${ }^{6,7}$

The Src family of kinases (cSrc, Lyn, Fyn, Yes, Lck, Blk, Hck) is a group of protein tyrosine kinases that function in the regulation of growth in eukaryotic cells. ${ }^{8}$ Activity of Src kinases is regulated by a variety of stimuli, and increased cSrc (or Src) kinase activity is a feature of several solid tumor types, ${ }^{8}$ including esophageal adenocarcinoma. Increased activity and expression of Src are also features of HGD-BE. ${ }^{9-12}$ Src targets a diverse number of proteins within the cell, one of which is the tumor suppressor p27. Responsible for regulation of the cell cycle, p27 has been found to be tyrosine phosphorylated by Src at 2 discrete sites (tyrosine 74 and 88), ${ }^{13}$ leading to increased p27 proteolysis, reduced p27 inhibitory binding of cyclin E-Cdk2, impaired p27 inhibition of cyclin D-cdk4 kinase activity, and correlation with p27 cytoplasmic localization 


\section{Abbreviations and Acronyms \\ $\mathrm{BE}=$ Barrett's esophagus \\ DMSO $=$ dimethyl sulfoxide \\ HGD = high-grade dysplasia}

in malignant tissues. ${ }^{13,14}$ The deregulation of p27 by Src results in increased cell proliferation. Small molecule inhibition of Src reverses these effects, restoring p27's ability to regulate the cell cycle and reduce growth. ${ }^{13} \mathrm{~A}$ retrospective analysis of samples from patients with HGD-BE has revealed increased p27 cytoplasmic localization (48\% of samples), ${ }^{12}$ suggesting involvement of p27 deregulation in the progression of $\mathrm{BE}$. On the basis of these previous observations in HGD-BE, we hypothesized that Src inhibition may have value in controlling HGD-BE and that Src alters the function of p27 in HGD-BE cells. To test this hypothesis, we investigated the effects of a small molecule inhibitor of the Src kinase (dasatinib) on the growth and regulation of p27 in immortalized BE cell lines.

\section{MATERIALS AND METHODS \\ Cell Lines and Patient Tissue}

The human Barrett's esophageal metaplastic (CP-A) and high-grade dysplastic (CP-D) human cell lines were obtained from American Type Culture Collection (Manassas, Va), maintained in recommended media and grown and cultured as previously described. ${ }^{15}$ Both cell lines harbor characteristic $\mathrm{BE}$ chromosomal abnormalities, increased ploidy, and p16/CDKN2 inactivation, but differ in p53 status (CP-A-p53 wt; CP-D p53 mutant). Although the CP-A cell line was established from a patient with metaplasia, a recent report has suggested caution in interpreting these cells as comparable to metaplasia because they have characteristics inconsistent with metaplastic BE. ${ }^{16}$ Patient tissues were obtained under a protocol approved by the institutional review board and managed by the Human Specimen Procurement Service (St Joseph's Hospital and Medical Center, Phoenix, Ariz).

\section{Drug Treatment}

Dasatinib (Selleck Chemicals, Houston, Tex) was dissolved in dimethyl sulfoxide (DMSO) to appropriate working concentrations with final DMSO less than $1 \%$. All controls were treated with an equivalent volume of DMSO diluted in culture media.

\section{Biochemical Analysis}

Total protein lysates from treated and untreated cells were isolated as described previously. ${ }^{17}$ Sample protein, 25 to $40 \mu \mathrm{g}$, was separated on $12 \%$ sodium dodecyl sulfate polyacrylamide gel electrophoresis gels and subsequently transferred to nitrocellulose at $4^{\circ} \mathrm{C}$ overnight. Immunoblotting was performed and developed as described previously. ${ }^{17}$ Antibodies specific to p-p27-S10, p-p27-T198, and p-p27-T157 were obtained from Abcam (Abcam, Cambridge, Mass). The p-p27-T187 antibody was acquired from LifeSpan Biosciences (LifeSpan Biosciences, Inc, Seattle, Wash). Antibodies to p27/Kip1, Src, phosphorylated Src (Y416), cyclin D, cyclin E, and cleaved Parp were obtained from Cell Signaling (Cell Signaling Technology, Boston, Mass).

\section{Quantitative Reverse Transcriptase Polymerase Chain Reaction}

Total RNA isolated with the RNeasy Mini Kit (QIAGEN Inc, Valencia, Calif) was used for first-strand cDNA synthesis using SuperScript III (Invitrogen, Carlsbad, Calif) and oligo(dT) (Invitrogen). Custom designed gene-specific primers (Invitrogen) were suspended to $10 \mu \mathrm{mol} / \mathrm{L}$, combined with LightCycler 480 SYBR Green I Master (Roche Applied Science, Indianapolis, Ind) and $2 \mu \mathrm{L}$ of the cDNA reaction. The samples were analyzed in triplicate on the LightCycler 480 (Roche Applied Science) and normalized to GAPDH. Fold change analysis was performed as previously described. ${ }^{18}$

\section{Cell Viability}

A total of 250 cells per well were plated on a 96-well plate with 8 replicates per treatment dose and allowed to attach overnight. Cells were treated with the appropriate concentration of dasatinib diluted in cell culture media for 24 hours. Viability was assessed with the CellTiter-Glo Luminescent Cell Viability Assay (Promega Corp, Madison, Wis) according to the manufacturer's instructions. Luminescence was read on the DTX880 Multimode Detector (Beckman Coulter Inc, Indianapolis, Ind). Viability of treated cells was determined relative to vehicletreated cells.

\section{Immunofluorescence and Brightfield Imaging}

$2 \times 10^{5}$ cells were seeded onto glass coverslips and allowed to attach overnight, followed by treatment with $100 \mathrm{nM}$ dasatinib. Drug was diluted from prepared stock and changed every 24 hours. Cells were fixed with $4 \%$ paraformaldehyde, permeabilized with $0.1 \%$ saponin/ phosphate-buffered saline, and blocked with CAS Block (Invitrogen). Primary (p27) and AlexaFluor488 conjugated secondary antibodies were diluted in $0.5 \%$ bovine serum albumin/phosphate-buffered saline and incubated overnight at $4^{\circ} \mathrm{C}$ or 2 hours at room temperature, respectively. Nuclei were stained and coverslips mounted onto glass slides with Prolong Gold with DAPI (Invitrogen) according to the manufacturer's instructions. Stained cells were visualized with a Zeiss 710 laser scanning confocal microscope (Carl Zeiss Microscopy, LLC, Thornwood, NY) equipped with a $63 \times / 1.4 \mathrm{NA}$ plan-apochromat objective. AlexaFluor488 (p27) was identified with $488 \mathrm{~nm}$ excitation and 505 to $525 \mathrm{~nm}$ emission. DAPI was identified with $405 \mathrm{~nm}$ excitation and 410 to $460 \mathrm{~nm}$ emission. Emission spectra were collected separately to minimize fluorophore overlap. Images were acquired with the confocal aperture set to 1 Airy unit and managed in Zen 2009 v 5.5.0.375 software (Carl Zeiss Microscopy, LLC). Brightfield images were viewed on a Zeiss Axiovert 200M (Carl Zeiss Microscopy, LLC, Thornwood, NY) and captured with an AxioCam ERc5s camera (Carl Zeiss Microscopy, LLC) at $10 \times$ magnification.

\section{Flow Cytometry}

To analyze the effects of dasatinib treatment on the cell cycle and apoptosis of BE cells, flow cytometry was performed on the Accuri C6 Flow Cytometer (BD Biosciences, San Jose, Calif) with a threshold at 80,000 and 20,000 events collected per sample. Cells $\left(1 \times 10^{6}\right.$ cells/100-mm dish) were allowed to attach overnight and treated with $100 \mathrm{nM}$ dasatinib for the indicated times. Assays were performed in triplicate and repeated. Cell-cycle analysis was conducted using propidium iodide to stain DNA content. RNA was eliminated with RNaseA. To determine apoptosis, phosphatidylserine externalization was used as an apoptotic marker and visualized using the Dead Cell Apoptosis Kit with Annexin V FITC (Invitrogen) per the manufacturer's recommended protocol. Phosphatidylserine was visualized by Annexin V FITC conjugation indicative of apoptosis. For comparison, cells were treated with camptothecin to induce apoptotic death to establish proper gating. Compensations were set to FL1-FL3 6\%, FL3-FL1 5\%. 


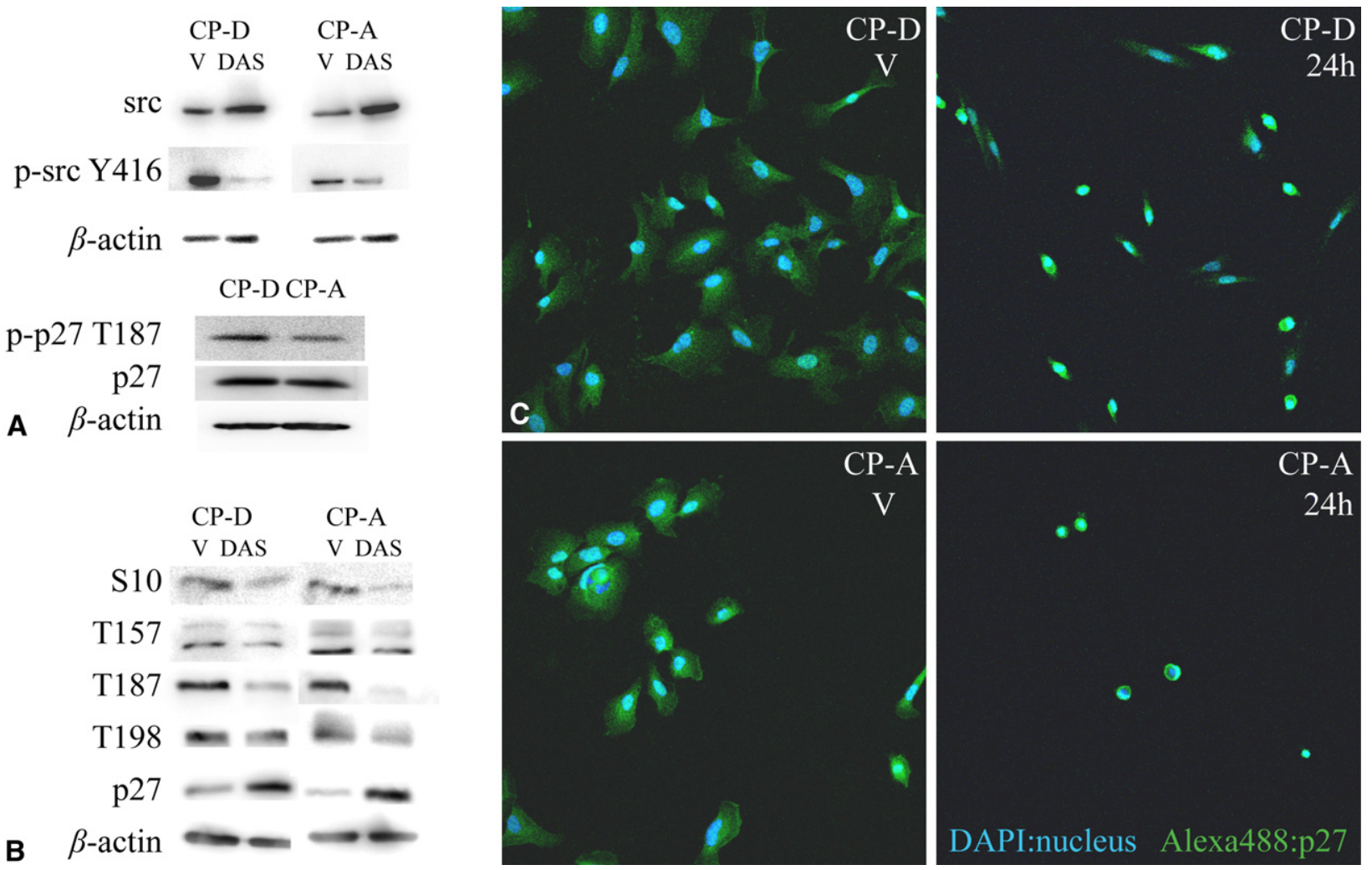

FIGURE 1. Dasatinib $(D A S)$ treatment reduces p27 phosphorylation and induces relocalization of p27/Kip1 to the nucleus. A, Top: CP-D and CP-A cells were treated for 4 hours with $100 \mathrm{nM}$ dasatinib or vehicle (DMSO). Total protein lysates were probed for active (phosphorylated) Src (p-src Y416). Dasatinib treatment results in reduced active Src. Bottom: CP-D and CP-A cells were probed with antibodies specific to phosphorylated-T187 p27 and p27. CP-D cells display increased basal phsophoryated-T187 p27, compared with CP-A cells. $\beta$-Actin was used as a loading control. Blot is representative of 3 independent experiments. B, Effect of dasatinib on p27 phosphorylation. CP-D and CP-A cells were treated with $100 \mathrm{~nm}$ (dasatinib) or vehicle (DMSO) for 24 hours. Total protein lysates were probed using antibodies specific to phosphorylated T187-p27, T157-p27, S10-p27, or total p27. Dasatinib treatment results in reduced p27 phosphorylation at all sites $(\mathrm{S} 10, \mathrm{~T} 157, \mathrm{~T} 187)$ with a corresponding increase in total p27 levels. $\beta$-Actin was used as a loading control. Blot is representative of 3 independent experiments. C, Immunofluorescence staining of p27 in CP-D and CP-A immortalized BE cell lines after a 24-hour treatment with $100 \mathrm{nM}$ dasatinib (dasatinib) or vehicle (DMSO) reveals relocalization of p27/kip1 (green) from the cytoplasm to the nucleus (blue). V, Vehicle (dimethyl sulfoxide).

\section{RESULTS}

\section{Dasatinib Reduces Src Activation in Immortalized Barrett's Esophagus Cells and Reverses p27 \\ Deregulation}

To investigate the effects of Src inhibition and p27 function in BE, we treated 2 immortalized BE cell lines established from patient tissue, one diagnosed as without dysplasia (CP-A) and one diagnosed with HGD (CP-D), ${ }^{15}$ with dasatinib, an inhibitor of Src kinase and Bcr-ABL oncogene, which is currently approved by the Food and Drug Administration as a second-line treatment for chronic myeloid leukemia. ${ }^{8}$ CP-D and CP-A cells were treated with 100 $\mathrm{nM}$ dasatinib and analyzed by immunoblot for Src activation with an antibody specific to tyrosine 416, an indicator of active Src. As shown in Figure 1, A, tyrosine phosphorylation at the 416 (p-Src Y416) site in both CP-D and CP-A cells was reduced after a 4-hour exposure to dasatinib, with the HGD CP-D cell line displaying increased active Src, relative to the metaplastic CP-A cell line. On the basis of the observed increased basal Src activity in CP-D and CP-A cells, we performed an immunoblot analysis of p27 for phosphorylation at threonine 187 (T187), a site shown to require Src tyrosine phosphorylation. ${ }^{13}$ Immunoblotting with an antibody specific to p27 T187-phosphorylation showed increased basal phosphorylation of p27 at the T187 site in CP-D cells, compared with CP-A cells (Figure 1, A, bottom). Treatment with dasatinib for 24 hours reduced p27 T187 phosphorylation and increased total p27 protein levels (Figure 1, B), consistent with observations that phosphorylation at $\mathrm{T} 187$ results in $\mathrm{p} 27$ proteosomal degradation. p27 is phosphorylated at multiple sites in addition to T187 (serine 10-S10, threonine 157-T157, threonine 198-T198), which can contribute to p27 deregulation. ${ }^{19} \mathrm{We}$ immunoblotted dasatinib-treated $\mathrm{BE}$ protein lysates with antibodies specific to phosphorylation at these sites ( 10 , T157, T198) to ascertain whether Src inhibition had any 
A

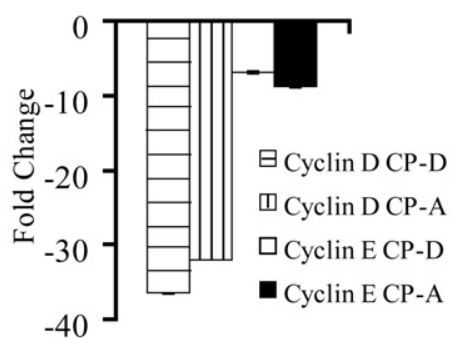

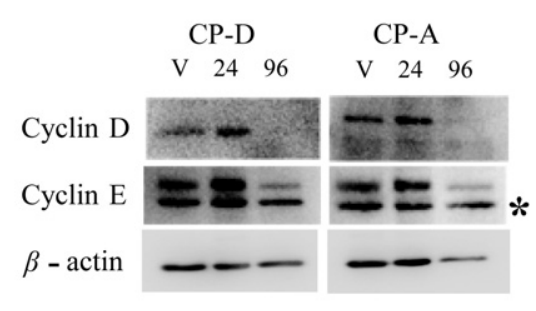

B

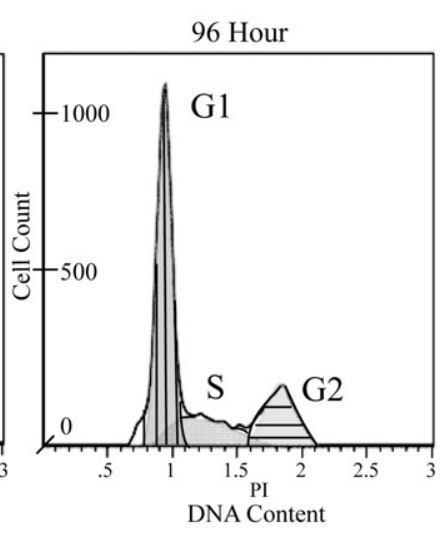

FIGURE 2. Dasatinib treatment results in cell-cycle arrest. A, Total RNA from vehicle-treated and dasatinib- (96 hours) treated CP-D and CP-A underwent expression analysis of cyclins D and E. Fold change is relative to expression of cyclin D or E in vehicle-treated cells. Data represent the mean of 2 independent experiments performed in duplicate. Bars represent standard error. B, Immunoblot analysis of cyclins D and E in CP-D and CP-A cells treated with $100 \mathrm{nM}$ dasatinib for 24 or 96 hours. Asterisk indicates nonspecific band migrating below cyclin E. $\beta$-Actin was used as a loading control. Blot is representative of 3 independent experiments. C, Cell-cycle analysis of CP-D cells after 96 hours of dasatinib (100 nM) treatment. CP-D cells were stained and analyzed as described in "Materials and Methods." Control represents cell-cycle profile of CP-D cells at the beginning of the experiment ( 0 hours). Data are the mean of 2 independent experiments.

effect on phosphorylation at those sites. Treatment with dasatinib resulted in decreases in phosphorylation at both $\mathrm{S} 10$ and T157 (Figure 1, B), sites shown to be involved in subcellular localization of $\mathrm{p} 27 .{ }^{19}$ Consistent with these findings, immunofluorescence of $\mathrm{p} 27$ showed that treatment with $100 \mathrm{nM}$ dasatinib induced the relocalization of p27 from the cytoplasm to the nucleus of both CP-D and CP-A cells after 24 hours (Figure 1, $C$, compare vehicle with 24 hours).

\section{Dasatinib Induces Cell-Cycle Arrest in Barrett's Esophagus Cell Lines}

Because treatment with dasatinib resulted in nuclear relocalization and increased protein levels of p27 in BE cell lines, we next investigated whether these changes correlated with alterations in the cell cycle. p27 directly associates and regulates cyclins $\mathrm{D}$ and $\mathrm{E}, 2$ proteins necessary for progression through the cell cycle. ${ }^{13,14}$ Expression analysis of CP-A and CP-D revealed that treatment with dasatinib resulted in considerable decreases in the mRNA of both cyclins D and E in CP-D and CP-A cell lines and particularly for cyclin D (Figure 2, A). To confirm these findings, protein levels of cyclins $\mathrm{D}$ and $\mathrm{E}$ were assessed in CP-D and CP-A BE cells treated with dasatinib for 24 and 96 hours. As shown in Figure 2, B, both cell lines displayed increases in protein levels of cyclins $D$ and $E$ after 24 hours of treatment. However, after 96 hours of dasatinib treatment, both BE cell lines showed dramatic decreases in cyclins D and E protein levels, consistent with the quantitative polymerase chain reaction data at 96 hours (Figure 2, $C)$. We next analyzed the DNA content and cell-cycle profile of CP-D cells treated with dasatinib by flow cytometry. As shown in Table 1 and Figure 2, C, CP-D cells displayed distinct changes in the percentages of cells in each cellcycle phase on treatment with dasatinib. Specifically, dasatinib treatment resulted in a decrease in $\mathrm{S}(37.60 \%$ at

TABLE 1. Dasatinib induces cell-cycle arrest at G1 in Barrett's esophagus cells

\begin{tabular}{lccccc}
\hline & $\mathbf{0}$ & $\mathbf{2 4}$ & $\mathbf{4 8}$ & $\mathbf{7 2}$ & $\mathbf{9 6}$ \\
\hline G1 & $37.60 \%$ & $45.41 \%$ & $64.48 \%$ & $69.04 \%$ & $73.27 \%$ \\
S & $34.99 \%$ & $26.3 \%$ & $25.13 \%$ & $24.94 \%$ & $22.77 \%$ \\
G2 & $37.13 \%$ & $33.67 \%$ & $20.95 \%$ & $14.52 \%$ & $14.36 \%$ \\
\hline
\end{tabular}

CP-D cells were treated with dasatinib for $24,48,72$, and 96 hours. Cell-cycle distribution was determined using propidium iodide as described in "Materials and Methods." Results are the mean of 2 independent experiments. 
A

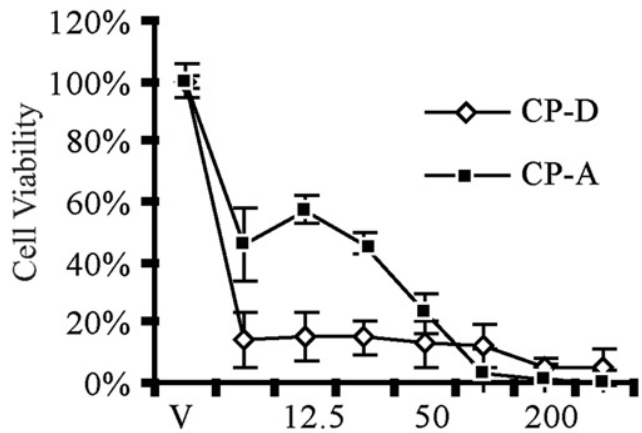

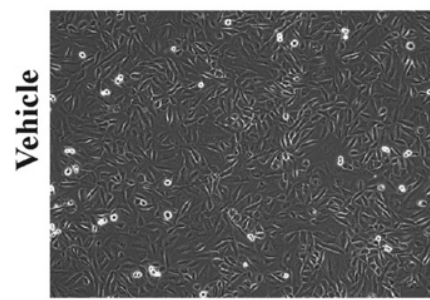

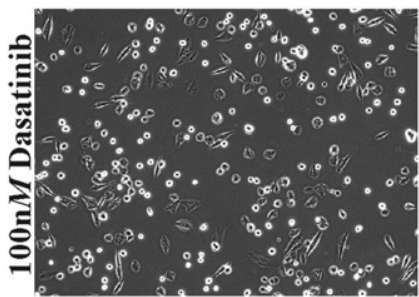

B

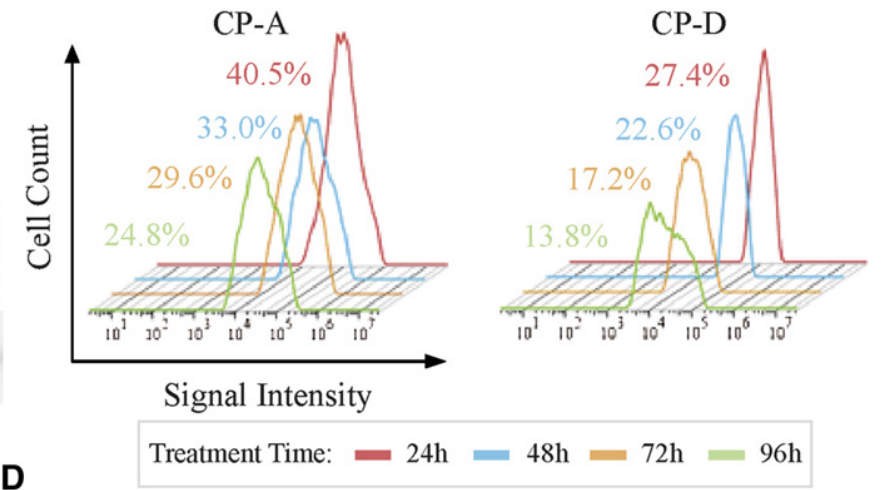

C

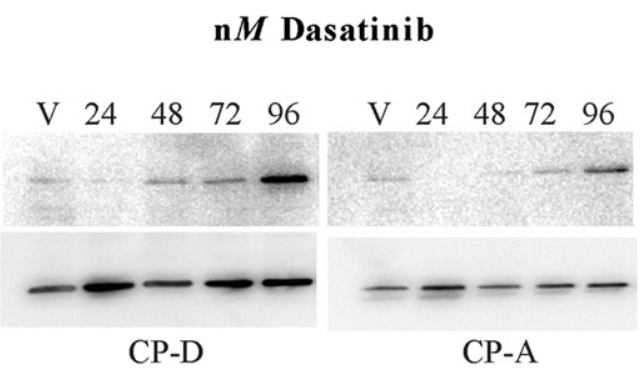

cleaved

Parp

$\beta$-actin

$$
\text { CP-D }
$$

CP-A

FIG for 24 hours. Viable cells were evaluated as described in "Materials and Methods." Note that CP-D cells (derived from high-grade dysplasia) display sensitivity to low nanomolar concentrations of dasatinib. Bars represent standard error. Data are the mean of 3 independent experiments. B, Brightfield images of CP-D cells treated with $100 \mathrm{nM}$ dasatinib for 72 hours. Note the increase in unattached, floating cells after 72 hours of treatment. C, Immunoblot analysis of CP-A and CP-D cells for the apoptotic marker, cleaved Parp. CP-A and CP-D cells were treated as in (B), and total protein lysates were collected at the indicated time points. $\beta$-Actin was used as a loading control. Blot is representative of 3 independent experiments. D, CP-D and CP-A cells were treated and analyzed as described in "Materials and Methods." Unfixed cells were collected at the indicated time points and evaluated for activation of apoptosis by annexin V staining using flow cytometry.

0 hours vs $22.77 \%$ at 96 hours) and $\mathrm{G} 2 / \mathrm{M}(37.13 \%$ at 0 hours vs $14.36 \%$ at 96 hours), phases with a corresponding increase in G1 (37.6\% at 0 hours vs $73.77 \%$ at 96 hours), compared with vehicle-treated cells. Similar results were observed in CP-A cells (data not shown).

\section{Prolonged Dasatinib Treatment Results in Apoptosis in Barrett's Esophagus Cell Lines}

We next tested the response of CP-D and CP-A cells to a range of dasatinib concentrations. After treatment for 24 hours, both CP-D and CP-A showed a significant decrease in the number of viable cells to all concentrations of dasatinib, compared with vehicle-treated cells (Figure 3, A). CP-D cells, derived from HGD, responded to lower concentrations of dasatinib compared with CP-A cells. We observed significant numbers of detached cells within the media of treated cells (Figure 3,B), suggesting that cells also could be activating apoptosis in response to dasatinib treatment. To investigate this possibility, dasatinib-treated CP-D and CP-A cells were analyzed by immunoblot using an antibody specific to cleaved Parp, a marker of apoptosis. Dasatinib treatment resulted in an increase in cleaved Parp at 48 hours, with maximal levels occurring after 96 hours of treatment (Figure 3,C). To confirm these findings, we assessed Annexin V staining, an early marker of apoptosis, after dasatinib treatment. Dasatinib treatment resulted in an increase in Annexin V staining after 24 hours in both cell lines, which remained throughout treatment (Figure 3,D).

\section{Phosphorylation of p27 in Barrett's Esophagus Patient Tissue}

On the basis of the observations of increased p27 phosphorylation by Src in CP-A and CP-D cells, we subsequently investigated p27 phosphorylation in patient tissue samples. Total protein lysates from patient biopsy samples with pathologic diagnoses of metaplastic $\mathrm{BE}(\mathrm{n}=4)$, HGD-BE $(\mathrm{n}=4)$, or esophagitis $(\mathrm{n}=3)$ were immunoblotted for p-T187 p27, p-T157 p27, p-S10 p27, total p27, and Src kinase. As shown in Figure 4, levels of Src were increased in patients with BE and HGD compared with patients with esophagitis, which is consistent with previous observations. $^{10,11}$ Phosphorylation of p27 at T187 was present at increased levels in all BE tissues and 2 of 4 HGD tissues, compared with esophagitis (Figure 4, A), 
A
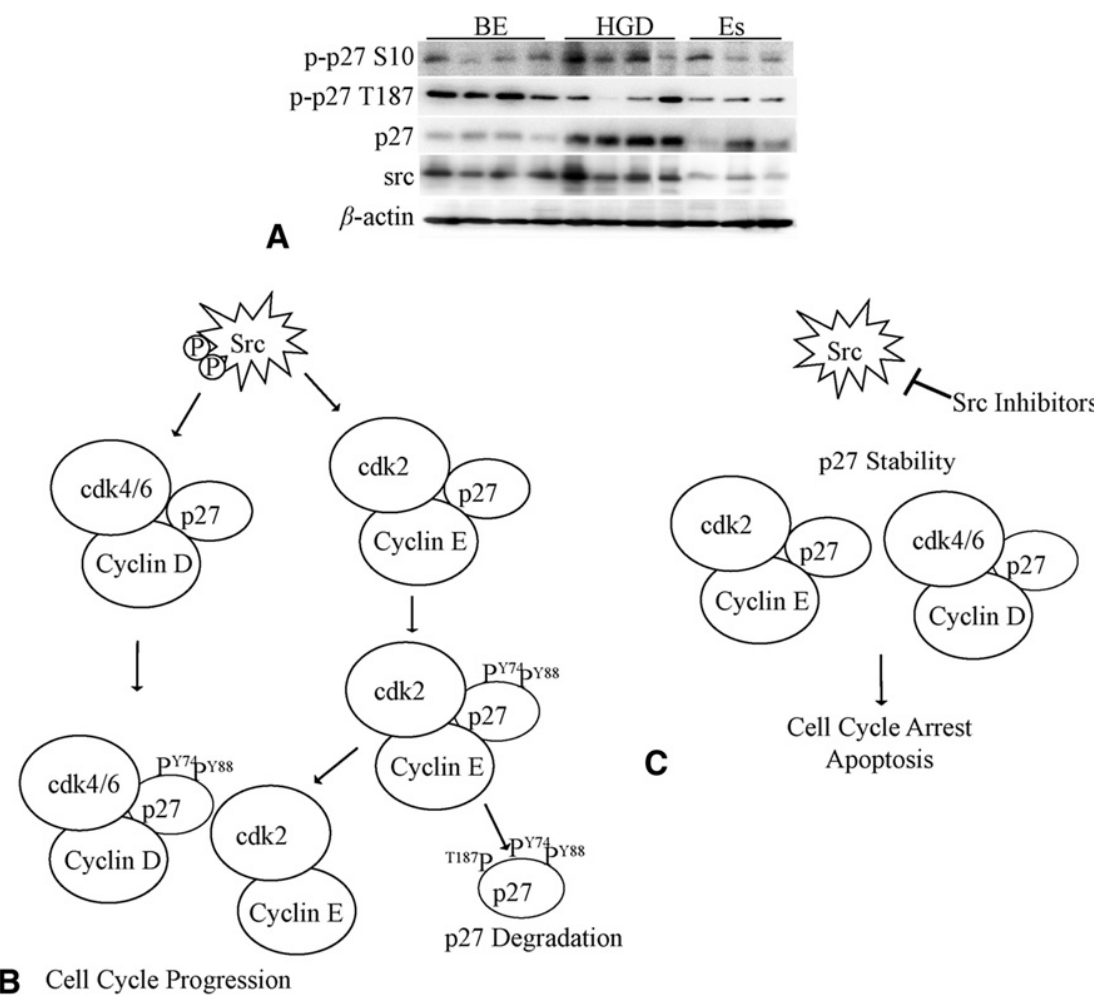

FIGURE 4. Abnormal phosphorylation of p27/Kip1 and increased expression of Src are present in biopsies of patients with Barrett's esophagus $(B E)$ and high-grade dysplasia $(H G D)$. A, Standard biopsies from patients diagnosed with metaplastic BE $(\mathrm{n}=4), \operatorname{HGD}(\mathrm{n}=4)$, or esophagitis $(E s)(\mathrm{n}=3)$ were used to isolate total protein as described in "Materials and Methods." Lysates were separated using standard procedures and immunoblotted with antibodies specific to p27 phosphorylated at T187 or S10. Lysates also were probed for total protein levels of Src and p27. $\beta$-Actin was used as a loading control. B and C, Potential model for dasatinib in BE cells. B, p27 phosphorylation by Src reduces p27 inhibitory functions and results in cell-cycle progression and p27 degradation. C, Inhibition of Src with small molecule inhibitors (eg, dasatinib) stabilizes p27 and allows p27 to inhibit function of cyclins E and $\mathrm{D}$ and induce cell-cycle arrest.

whereas 3 of 4 HGD-BE samples showed increased S10 phosphorylation compared with $\mathrm{BE}$ and esophagitis (Figure 4). Tissues of patients with HGD demonstrated increased total levels of p27 compared with BE (Figure 4), shown to be a consequence of p27 present throughout the entire BE intestinal gland in HGD and restriction of p27 to superficial regions of the BE gland. ${ }^{12}$ Analysis of p-T157 was inconclusive, with little to no staining in any of the tissues (data not shown).

\section{DISCUSSION}

The present study explored the effects of a Food and Drug Administration-approved, small molecule inhibitor of the Src kinases, dasatinib, on BE cells. Treatment of immortalized BE cell lines (metaplasia and HGD) in vitro with dasatinib results in reduced proliferation, cell-cycle arrest, and apoptosis. These observations correlate with inhibition of Src, p27 nuclear relocalization, increased protein levels of p27, and reduction of p27 phosphorylation. These findings collectively suggest that dasatinib and other Src kinase inhibitors may have potential as chemopreventive agents for HGD$\mathrm{BE}$ and whose efficacy can be assessed by evaluation of $\mathrm{p} 27$.
Preclinical studies indicate that dasatinib and other Src inhibitors have substantial chemotherapeutic activity in malignant cells, inhibiting cell-cycle progression, proliferation, and metastasis, and activating apoptosis. ${ }^{8}$ The current study found that dasatinib treatment results in cell-cycle arrest after 96 hours of treatment (Figure 2, $C$, and Table 1), increases cyclins D and E after 24 hours of treatment, and decreases cyclin D and E expression at 96 hours (Figure 2, $A$ and $B$ ) and apoptosis (Figure 3 ) in premalignant BE cells. Further, these characteristics also are found with p27-induced growth arrest ${ }^{20-22}$ and forced p27 expression in cells. ${ }^{23,24}$ These findings suggest that the antineoplastic effects of dasatinib observed within $\mathrm{BE}$ cell lines might be due to reversal of Src-mediated deregulation of p27 and support a potential model for the effects of dasatinib in our BE cell lines (Figure 4, $B$ and $C$ ). Evidence indicates that tyrosine phosphorylation of p27 by Src permits T187-phosphorylation of p27 by cyclin E-cdk2, promoting disassociation of p27 from the cyclin E-cdk2 complex and proteosomal degradation, ${ }^{13}$ as well as being necessary for activation of the cyclin D-cdk $4 / 6$ complex $^{14}$ (Figure 4, B). Combined, these events result in cell-cycle 
progression. Inhibition of Src by dasatinib stabilizes p27 protein levels and nuclear relocalization, allowing p27 to inhibit progression of the cell cycle and induce cell-cycle arrest (Figure 4,C). However, because of the broad effects of Src within eukaryotic cells, ${ }^{8}$ as well as dasatinib's ability to functionally inhibit other tyrosine kinases, it is possible that dasatinib could be producing the observed responses via inhibition of an Src-dependent or Src-independent pathway. Our observations of reduction in other p27 phosphorylation sites may reflect this possibility because phosphorylation at these sites (S10, T157, T198) has been linked to activity of other kinases. ${ }^{19}$ Thus, it will be important in future investigations to definitively implicate p27 as a mediator of dasatinib's antineoplastic effects in BE cells, a topic that is currently being pursued in our laboratory.

Although dasatinib has displayed minimal efficacy in late-stage solid tumors as a monotherapy, ${ }^{8}$ our evidence suggests that dasatinib (even at low doses) could have benefits for control of HGD-BE, a premalignant lesion. The current state of treatment for HGD-BE focuses on local ablation or surgical removal of the lesion. ${ }^{5}$ Although efficacious, these procedures are expensive and often require multiple treatments. In addition, the invasive nature of these procedures can be unattractive to patients. Development of chemopreventive strategies that limit and perhaps even eradicate the HGD-BE lesion would have considerable patient benefit, providing a safe, noninvasive method to control their BE. Such an approach is not unfounded, as evidenced by the Aspirin and Esomeprazole Chemoprevention (AspECT) in BE trial currently ongoing in Europe and smaller studies investigating the chemopreventive potential of aspirin and other nonsteroidal anti-inflammatory drugs. ${ }^{1,6}$ However, despite the moderate efficacy of nonsteroidal anti-inflammatory drugs in limiting BE progression, ${ }^{1,6}$ the cardiovascular, gastrointestinal, and renal side effects of these agents have limited their clinical application ${ }^{25}$ and led to the pursuit of alternative modalities.

\section{CONCLUSIONS}

Our in vitro studies with dasatinib are an early preliminary step toward an alterative chemopreventive agent for HGD-BE. Our observations of reduced growth and apoptosis at low doses of dasatinib could indicate that patients with HGD-BE may benefit from dasatinib at low doses or short durations of treatment. Further, because our studies show the occurrence of Src-dependent p27 phosphorylation and increased Src expression in HGD$\mathrm{BE}$, evaluation of $\mathrm{p} 27$ localization or $\mathrm{p} 27$ phosphorylation would be a useful marker for Src inhibition in both preclinical and clinical studies. However, despite the beneficial effects of dasatinib in BE cell lines, substantial studies are necessary before dasatinib can be applied clinically. Specifically, testing the efficacy of low-dose dasatinib in controlling BE progression, as well as developing dosing and treatment regimens in surgical rodent models of BE, will be important before attempting patient studies. Such studies are critical, because a chemopreventive strategy for HGD-BE would beneficially affect early adenocarcinoma by providing multiple avenues for early treatment.

\section{References}

1. Reid BJ, Li X, Galipeau PC, Vaughan TL. Barrett's oesophagus and oesophageal adenocarcinoma: time for a new synthesis. Nat Rev Cancer. 2010;10: 87-101.

2. Pohl H, Welch HG. The role of overdiagnosis and reclassification in the marked increase of esophageal adenocarcinoma incidence. J Natl Cancer Inst. 2005;97: 142-6.

3. Reid BJ, Levine DS, Longton G, Blount PL, Rabinovitch PS. Predictors of progression to cancer in Barrett's esophagus: baseline histology and flow cytometry identify low- and high-risk patient subsets. Am J Gastroenterol. 2000;95: 1669-76.

4. Yousef F, Cardwell C, Cantwell MM, Galway K, Johnston BT, Murray L. The incidence of esophageal cancer and high-grade dysplasia in Barrett's esophagus: a systematic review and meta-analysis. Am J Epidemiol. 2008;168: 237-49.

5. Spechler SJ, Sharma P, Souza RF, Inadomi JM, Shaheen NJ. American Gastroenterological Association medical position statement on the management of Barrett's esophagus. Gastroenterology. 2011;140:1084-91.

6. Galipeau PC, Li X, Blount PL, Maley CC, Sanchez CA, Odze RD, et al. NSAIDs modulate CDKN2A, TP53, and DNA content risk for progression to esophageal adenocarcinoma. PLoS Med. 2007;4:e67.

7. Chang CL, Lao-Sirieix P, Save V, De La Cueva Mendez G, Laskey R, Fitzgerald RC. Retinoic acid-induced glandular differentiation of the oesophagus. Gut. 2007;56:906-17.

8. Puls LN, Eadens M, Messersmith W. Current status of SRC inhibitors in solid tumor malignancies. Oncologist. 2011;16:566-78.

9. Iravani S, Zhang HQ, Yuan ZQ, Cheng JQ, Karl RC, Jove R, et al. Modification of insulin-like growth factor 1 receptor, $\mathrm{c}-\mathrm{Src}$, and Bcl-XL protein expression during the progression of Barrett's neoplasia. Hum Pathol. 2003;34:975-82.

10. Jankowski J, Coghill G, Hopwood D, Wormsley KG. Oncogenes and oncosuppressor gene in adenocarcinoma of the oesophagus. Gut. 1992;33:1033-8.

11. Kumble S, Omary MB, Cartwright CA, Triadafilopoulos G. Src activation in malignant and premalignant epithelia of Barrett's esophagus. Gastroenterology. 1997; 112:348-56.

12. Singh SP, Lipman J, Goldman H, Ellis FH, Aizenman L, Cangi MG, et al. Loss or altered subcellular localization of p27 in Barrett's associated adenocarcinoma. Cancer Res. 1998;58:1730-5.

13. Chu I, Sun J, Arnaout A, Kahn H, Hanna W, Narod S, et al. p27 phosphorylation by Src regulates inhibition of cyclin E-Cdk2. Cell. 2007;128:281-94.

14. James MK, Ray A, Leznova D, Blain SW. Differential modification of p27Kip1 controls its cyclin D-cdk4 inhibitory activity. Mol Cell Biol. 2008 28:498-510.

15. Palanca-Wessels MCA, Klingelhutz A, Reid BJ, Norwood TH, Opheim KE, Paulson TG, et al. Extended lifespan of Barrett's esophagus epithelium transduced with the human telomerase catalytic subunit: a useful in vitro model. Carcinogenesis. 2003;24:1183-90.

16. Kosoff RE, Gardiner KL, Merlo LM, Pavlov K, Rustgi AK, Maley CC. Development and characterization of an organotypic model of Barrett's esophagus. J Cell Physiol. 2012;227:2654-9.

17. Inge LJ, Coon KD, Smith MA, Bremner RM. Expression of LKB1 tumor suppressor in non-small cell lung cancer determines sensitivity to 2-deoxyglucose. J Thorac Cardiovasc Surg. 2009;137:580-6.

18. Fleige S, Walf V, Huch S, Prgomet C, Sehm J, Pfaffl MW. Comparison of relative mRNA quantification models and the impact of RNA integrity in quantitative real-time RT-PCR. Biotechnol Lett. 2006;28:1601-13.

19. Chu IM, Hengst L, Slingerland JM. The Cdk inhibitor p27 in human cancer: prognostic potential and relevance to anticancer therapy. Nat Rev Cancer. 2008;8:253-67.

20. Craig C, Wersto R, Kim M, Ohri E, Li Z, Katayose D, et al. A recombinant adenovirus expressing p27Kip1 induces cell cycle arrest and loss of 
cyclin-Cdk activity in human breast cancer cells. Oncogene. 1997;14: 2283-9.

21. Sgambato A, Zhang YJ, Ciaparrone M, Soh JW, Cittadini A, Santella RM, et al. Overexpression of p27Kip1 inhibits the growth of both normal and transformed human mammary epithelial cells. Cancer Res. 1998;58:3448-54.

22. St Croix B, Sheehan C, Rak JW, Florenes VA, Slingerland JM, Kerbel RS. E-Cadherin-dependent growth suppression is mediated by the cyclin-dependent kinase inhibitor p27(KIP1). J Cell Biol. 1998;142:557-71.
23. Wang X, Gorospe M, Huang Y, Holbrook NJ. p27Kip1 overexpression causes apoptotic death of mammalian cells. Oncogene. 1997;15:2991-7.

24. Katayose Y, Kim M, Rakkar AN, Li Z, Cowan KH, Seth P. Promoting apoptosis: a novel activity associated with the cyclin-dependent kinase inhibitor p27. Cancer Res. 1997;57:5441-5.

25. Cuzick J, Otto F, Baron JA, Brown PH, Burn J, Greenwald P, et al. Aspirin and non-steroidal anti-inflammatory drugs for cancer prevention: an international consensus statement. Lancet Oncol. 2009;10:501-7. 